\title{
Novel insights provided by spectral-domain coherent tomography in pediatric ophthalmology
}

\author{
Marc M. Abitbol
}

Received: 22 August 2014 / Accepted: 27 August 2014 / Published online: 14 September 2014

(C) Springer-Verlag Berlin Heidelberg 2014

Tilted disk syndrome (TDS) is observed in approximately $1 \%$ to $2 \%$ of the population. This percentage appears to be highly variable according to the clinical and imaging features retained for establishing the accurate diagnosis. It is of paramount importance to distinguish isolated tilted optic disk from TDS. This syndrome has a number of alternative nomenclatures such as the nasal fundus ectasia syndrome, inversion of the optic disk, inverse myopia, and dysversion of the optic disk. TDS may be a variant of colobomatous defects. The definition of TDS has often been inaccurate in many studies which did not take account of the antero-posterior tilt and torsion of the optic disk. This so-called torsion corresponds to the rotation of the optic nerve around its sagittal axis. Additionally, until now there was neither an objective index of tilt nor any objective index of rotation. Surrogate indexes were proposed based on the elliptic form of the optic disk. The ratio of the minimal diameter and the maximal diameter of the optic disk or the inverse ratio have been used for evaluating the tilt. Normal optic disks are minimally oval, with the vertical diameter slightly longer than the horizontal. Optic disk torsion can be viewed and measured in twodimensional space. The longest diameter usually falls within $15^{\circ}$ of the vertical meridian; axes beyond $15^{\circ}$ are, by definition, torted. Most tilted disks appear to rotate excessively about the sagittal axis of the optic nerve, such that the longest diameter of the disk falls outside this $30^{\circ}$ boundary. This apparent rotation is often accentuated by the orientation of retinal vessels that seem to turn in a direction to compensate

\footnotetext{
M. M. Abitbol ( $\bowtie)$

Department of Ophthalmology, Necker-Enfants Malades University

Hospital of Paris, Paris Descartes University, 149 rue de Sèvres,

75015 Paris, France

e-mail: marc.abitbol@nck.aphp.fr

M. M. Abitbol

e-mail: marc.abitbol@parisdescartes.fr
}

for the "twisted" position of the optic disk [1]. The most bilateral clinical features retained for establishing clinically the diagnosis of TDS are the following ones : infero-nasal disk tilting, frequent superiorly elevated margins of the optic nerve head because of the tilting effect - this aspect can at times mimick the appearance of papilledema, inferonasal or inferior crescent or conus-which corresponds to the inferior peripapillary area devoid of choroid and retinal pigment epithelium and is often designated as Fuchs coloboma, situs inversus - characterized by the initial nasal orientation of the temporal retinal vessels on the optic disk followed secondarily by a temporal orientation, in contrast to the normal temporal orientation of these vessels from the outset, myopia ( $>1 \mathrm{D})$, astigmatism ( $>1 \mathrm{D})$, hypopigmented inferior fundus ectasia of 1-4 D (most eyes displaying this ectasia have associated superotemporal visual field defects), and decreased vision BVCA of $20 / 25$ to $20 / 50$. Bilaterality of these clinical features is observed in $75 \%$ of the cases. A thorough examination of patients with tilted disk includes refraction, dilated fundus examination, and visual field testing [2]. Familiarity with the spectrum of ophthalmoscopic appearance and the clinical manifestations of tilted disks may be the most critical factors in avoiding misdiagnosis. At times, neuroimaging may be necessary to arrive at the correct diagnosis. It was considered until very recently that until normative data are validated for tilted disks, the role of new imaging technologies, such as spectral-domain optical coherence tomography (SD OCT) for the optic nerve head, would remain limited. However, recent studies published in this journal and others are changing the landscape of pediatric ophthalmology in general and that of optic disk congenital abnormalities. They are helping to provide preliminary validated normative data and suggest new pathogenic hypotheses capable of explaining some crucial features of TDS and other congenital optic disk abnormalities. Despite some limitations, a pioneering study of a cohort of normal children and a cohort of children affected by TDS, 
using both SD OCT and annual visual field testing, led to the discovery that $50 \%$ of the children affected by TDS presented some amount of visual field defect at baseline, which completely regressed after maximal myopic correction. It is interesting to note that most of these children with baseline visual field defects displayed a significant nasal herniation of the nerve fibers unequivocally visualized by SD OCT. At the 1-year follow-up, a visual field defect persisted in the TDS affected patients; however, at this time point, $18.4 \%$ of the TDS-affected children who presented a reduction in thickness of the nerve fibers inside the Bruch's membrane protrusion detected by SD OCT also had a persistent visual field defect despite myopic correction. Although the mechanism causing the visual field defects that were not improved by increased myopic correction has not been determined, it is possible that it is due to the severe bending of the retinal nerve fiber tissue, which would impair axonal flow. This seminal observation should lead to the establishment of a multicentric study of TDS patients using the latest ophthalmic and neuroimaging techniques [3]. Another important study highlighted the importance of SD OCT and swept-source optical coherence tomography (SS OCT) in the differential diagnosis of congenital excavated optic disc anomalies. Morning glory syndrome, optic disc coloboma, and peripapillary staphyloma were respectively characterized by the presence of a preretinal tractional membrane, inferiorly decentered excavation, and an excavation deeper than that observed in morning glory syndrome and optic disc coloboma [4]. These reports show unequivocally that no modern department of pediatric ophthalmology can take care properly of children affected by congenical ophthalmic diseases or inherited eye diseases without including in the examination of these patients the results provided by the most recent techniques of optical coherence tomography, especially in university hospitals.

\section{References}

1. Witmer MT, Margo CE, Drucker M (2010) Tilted optic disks. Surv Ophthalmol 55(5):403-428

2. Brown CG, Brown MM (2014) Congenital abnormalities of the optic disk. In: Nelson BN, Olitsky SE (eds) Harley's pediatric ophthalmology, 6th edn. Lippincott Williams \& Wilkins, Philadelphia, pp 326327

3. Pichi F, Romano S, Villani E, Lembo A, Gilardoni F, Morara M, Ciardella AP, Ohno-Matsui K, Nucci P (2014) Spectral-domain optical coherence tomography findings in pediatric tilted disc syndrome. Graefes Arch Clin Exp Ophthalmol July 20 [Epub ahead of print]. doi:10.1007/s00417-014-2701-8

4. Lee KM, Woo SJ, Hwang JM (2014) Evaluation of congenital excavated optic disc anomalies with spectral-domain and swept-source optical coherence tomography. Graefes Arch Clin Exp Ophthalmol Jun 7 [Epub ahead of print]. doi:10.1007/s00417-014-2680-9 